\title{
Trabeculectomy Improves Vessel Response Measured by Dynamic Vessel Analysis (DVA) in Glaucoma Patients
}

\author{
J. Michael Selbach ${ }^{*}, 1,2$, Maurice Schallenberg ${ }^{2}$, Sebastian Kramer $^{2}$, Gerasimos Anastassiou ${ }^{1,2}$, \\ Klaus-Peter Steuhl' ${ }^{2}$, Walthard Vilser ${ }^{3,4}$ and Stephan Kremmer ${ }^{1,2}$
}

\author{
${ }^{I}$ Department of Ophthalmology, Evangelische Kliniken Gelsenkirchen, Munckelstr. 27, 45879 Gelsenkirchen, Germany \\ ${ }^{2}$ Department of Ophthalmology, University of Duisburg-Essen, Hufelandstr. 55, 45122 Essen, Germany \\ ${ }^{3}$ Institute of Biomedical Engineering and Informatics, Ilmenau University of Technology, Ilmenau, Gustav-Kirchhoff- \\ Str.2, Germany \\ ${ }^{4}$ Imedos Systems UG, Jena, Am Naßtal 4, Germany
}

\begin{abstract}
Purpose: To determine the effects of surgical IOP reduction (trabeculectomy) on retinal blood flow parameters in glaucoma patients using Dynamic Vessel Analysis (DVA).

Methods: 26 eyes of 26 patients with progressive primary open-angle glaucoma (POAG) despite maximal topical therapy were examined before and after trabeculectomy. The responses of the retinal vessels to flickering light provocation were measured with DVA the day before surgery and 4 to 6 weeks after trabeculectomy. Between 3 and 4 weeks before surgery all local therapies were stopped and a systemic therapy with acetazolamide and conservative free topic steroidal eye drops was started.

Results: In 19 patients (73\%), an inadequate response to the flicker stimulation was measured preoperatively. In these patients, the maximum dilation of arteries and veins was reduced significantly as compared to healthy eyes. In this group, the maximum dilation of the arteries following the flicker provocation improved from $1.4 \%$ before to $3.8 \%$ following trabeculectomy $(\mathrm{p}<0.01)$. In retinal veins, this parameter increased from $3.1 \%$ to $4.6 \%(\mathrm{p}<0.05)$. In the 7 patients whose arterial and venous reactions to flickering light provocation preoperatively did not differ from healthy eyes, there was no significant change after surgery. The initial baseline values of arteries and veins (MU) did not deviate significantly in both groups.

Conclusion: POAG patients with progressive disease and impaired vascular regulation profit from IOP lowering trabeculectomy concerning vascular reactivity and dilative reserve, indicating a possible improvement of retinal perfusion following effective IOP control. Future studies with long-term follow-up must determine the clinical importance of these findings for the treatment of glaucoma patients.
\end{abstract}

Keywords: Dynamic vessel analyzer (DVA), glaucoma, IOP, retinal autoregulation, trabeculectomy, vascular dysregulation.

\section{INTRODUCTION}

For many glaucoma patients, an elevated intraocular pressure (IOP) is not regarded as the sole pathogenetic factor. It has been proposed that vascular dysfunction contributes to the pathogenesis of glaucoma, a disease that often is associated with other manifestations of vascular dysfunction such as cerebrovascular and systemic cardiovascular disturbances [1].

Vascular dysregulation in the vessels of the posterior segment of the eye is manifested as an inadequate constriction or vasodilatation of retinal arteries in response to altered metabolic demands. Besides lacking such an adequate response, functional abnormalities like a reduced

*Address correspondence to this author at the Department of Ophthalmology, Evangelische Kliniken Gelsenkirchen, Munckelstr. 27, 45879 Gelsenkirchen, Germany; Tel: 0049 - 209 - 37261;

Fax:0049-209-378555; E-mail: michael.selbach@arcor.de blood flow velocity and increased resistance in retinal arteries have been demonstrated to be associated with glaucoma, particularly NTG. A clinical hallmark, relatively easy to detect by ophthalmoscopy, of such an altered endothelial function are optic disc haemorrhages which are associated with disturbances of ocular blood flow and autoregulation in glaucoma [2].

While vascular factors in glaucoma have been identified and are widely discussed, glaucoma therapy focuses predominantly on IOP reduction while attempts to increase blood flow, autoregulation and to ease the effects of vascular dysfunction presently play only a secondary role in the management of glaucoma patients. Currently it is difficult to measure intraocular blood supply and perfusion [3]. The Dynamic Vessel Analysis (DVA) has the potential to correctly measure the vessel diameter as the most important factor $\left(\mathrm{r}^{4}\right)$ of blood circulation according to the HagenPoiseuille law. Autoregulative dilation of retinal arterial 
vessels for instance can compensate for reduced retinal perfusion pressure in a defined range - the dilation reserve of arteries. One of the assumed mechanisms for that is the flow induced autoregulation based on intact vascular endothelial function. This function can be tested by measuring the vessel dilation caused by flickering light which in healthy eyes causes arteries and veins to dilate [4]. Vascular dysfunction may lead to reduced dilation reserve.

Nguyen et al. (2009) have demonstrated the high reproducibility of DVA for repeated measures over a short period of time on a study group of 33 healthy subjects. The authors reached the conclusion that such measurements may allow non-invasive quantification of endothelial function to study its association with systemic and ocular diseases [5]. A general overview of the use of DVA in research and in a clinical setting and informed guidelines for its use have recently been given by Garhofer et al. [6]. With regards to another recent publication, DVA holds promise to become a valuable tool of vessel assessment in the future [7].

Several studies using DVA on the haemodynamics in diabetic patients pointed out abnormally reduced vessel responses to stimulation as a possible underlying cause for well-known clinical entities like e.g. diabetic retinopathy [810]. This diminished response decreases, according to Schiel et al. [9], with increasing stages of retinopathy. Interestingly, the response in a group of diabetics was already reduced before clinical appearance of retinopathy [10]. By means of DVA technology, Mehlsen et al. [11] could not observe a normalizing effect of antihypertensive drugs such as calcium channel blockers and angiotensin converting enzyme inhibitors on retinal autoregulation. The authors concluded that it might take a longer time to improve autoregulation than to reduce the arterial blood pressure.

Rickenbacher et al. [12] have recently proven that otherwise healthy, vasospastic individuals demonstrated an altered reaction of the retinal vasculature to flickering light. This finding has possible clinical implications since vascular dysregulations are considered to be a major risk factor for a number of eye diseases, most notably for normal-tension glaucoma (NTG).

The fact that in glaucoma retinal vessels display abnormalities in their autoregulation has been established in an earlier study with a Retinal Vessel Analyzer by Garhöfer et al. [13]. Flickering light-induced vasodilation in retinal veins was significantly diminished in glaucoma patients as compared with healthy volunteers. The authors concluded that these results indicate impairment in the regulation of retinal vascular tone in patients with early glaucoma, independent of antiglaucoma medication.

Lowering the intraocular pressure, however, is not only regarded as a way to ease "mechanical" stress on retinal ganglion cells but also as an intervention to improve ocular perfusion by increasing perfusion pressure for microcirculation. Surgical intervention to lower IOP is widely considered the method of choice when a substantial IOP drop is required. Despite the development of new surgical techniques such as deep sclerectomy, canaloplasty, stents and valves, trabeculectomy is still a widely performed IOP-lowering procedure.
The rational of our study is that in glaucoma patients, the high IOP causes a reduced or exhausted dilation reserve of retinal vessels in response to flickering light. After treatment, we expect an improvement of the arterial flicker response.

Thus the aim of our study is to show that a surgical IOP reduction (trabeculectomy) improves vascular autoregulation as an important factor in preventing the progress of glaucomatous damage.

\section{MATERIALS AND METHODOLOGY}

We examined 26 eyes of 26 patients with primary openangle glaucoma (POAG). The responses of the retinal vessels to flickering light provocations were measured before and after trabeculectomy. The DVA examinations took place the day before surgery and 4 to 6 weeks after the intervention.

Exclusion criteria for this study were previous glaucoma surgery, other eye diseases (such as macular degeneration; only a mild cataract was allowed), diabetes mellitus and arterial hypertension (defined as a median blood pressure of more than $160 / 90 \mathrm{~mm} \mathrm{Hg}$ ). Therefore none of our patients received any systemic antidiabetic or antihypertensive medication. The study was performed in accordance to the declaration of Helsinki on good medical practice. Patients gave their informed consent.

The indication for surgery was based on the progress of glaucomatous damage (visual fields and optic disc) and/or elevated intraocular pressure despite a maximum local therapy with 3-4 different kinds of eye drops. Preoperatively, all patients received systemic acetazolamide for 3-4 weeks and preservative-free topical dexamethasone whereas topical antiglaucomatous medication was stopped for this period of time. Postoperatively, preservative-free topical dexamethasone and ofloxacine were administered. Trabeculectomy was performed in a usual manner with topical application of mitomycin C $0.02 \%$ to prevent scarring of the bleb. Postoperatively no early needling of the bleb was necessary. However, in some cases a suture lysis was done during the first 2 weeks.

The Vessel Analysis with the DVA (Dynamic Vessel Analyzer; Imedos Systems UG, Jena) is a noninvasive method to examine retinal vessel parameters based on vessel diameter measurements to describe the vascular function (autoregulative vessel responses due to stimulation or provocation of the microcirculation). The DVA was developed to analyze retinal vessels with diameters between 60 and $200 \mu \mathrm{m}$. We used a Dynamic Vessel Analyzer to investigate the arterial and venous flicker response before and after treatment.

The device takes video image sequences of the fundus under green monochromatic light using a special CCD camera fitted to a retinal camera. The images are digitized and analyzed online by a computer which is part of the DVA system. Along each selected vascular segment of up to $2 \mathrm{~mm}$ in length, the vessel's diameter is determined image-byimage in real time. The possible time resolution is 25 measurements per second, the spatial resolution $18 \mu \mathrm{m}$ in the vessel direction and $<1 \mu \mathrm{m}$ in the measurement direction [14]. 
The measuring principle of the DVA is basically less a direct assessment of the vessel's width but rather a measurement of the diameter of the column consisting of red blood cells streaming in the artery or the vein. These blood cells absorb a certain amount of the light emitted by the fundus camera and which is reflected by different retinal layers. Measurement algorithms of the DVA assess vessel diameters from brightness profiles and compensate for disturbances like shadowing structures and reflections on the vessel surface. A properly dilated pupil is essential for the examination's validity. The observer defines a measurement area by dragging a "region of interest" over a chosen vessel that is being watched in real time. The measurement is then started by a mouse click and the arterial and venous vessel diameters of the selected vessels are recorded [15].

During diameter measurements, flickering light stimulation of the retina is performed. The flickering light induces metabolic demands of retinal tissue causing diameter changes of the retinal vessels. This behaviour is based on the so called neurovascular coupling and is recorded over time for all segments of the selected vessel. The vessel diameter will be continuously assessed over $350 \mathrm{~s}$. After recording of the baseline for $50 \mathrm{~s}$, three flickering cycles will follow. These flickering cycles consist of $20 \mathrm{~s}$ flicker followed by a resting period of $80 \mathrm{~s}$. In order to carry out the flickering period a stimulation unit will interrupt the green illumination light in an image synchronic way (one image bright, one image dark, frequency $12.5 \mathrm{~Hz}$, bright/ dark contrast 25:1). The measuring will always be carried out during the bright image period of the flickering light. Healthy vessels react to flickering light with dilation. In the recovery period the diameter returns to the baseline with an overshooting period (temporary vasoconstriction). The measuring program determines by signal averaging of 3 measuring cycles (flicker phase and resting period) the mean vessel response to flickering light relative to the base line. As parameters the maximum flicker dilation and the maximum constriction will be determined.

A muscarinic antagonist was used for pupil dilation, vasoactive drugs such as caffeine or nicotine were avoided.

After the measurement procedure, the software of the DVA provides several vessel parameters:

- $\quad$ an initial baseline value for the width of an artery or a vein is given in measuring units (MU); $1 \mathrm{MU}=1 \mu \mathrm{m}$ if the eye is fulfilling the conditions of the Gullstrand normal eye. For many eyes this is not the case and inter-individual aberrations may be expected. Therefore, no absolute values are given but the term measuring units (MU) is introduced allowing an intraindividual comparison.

- $\quad$ maximum dilation (as \% of the initial baseline) of an artery or vein during flicker stimulation

\section{maximum constriction after flicker stimulation}

peak amplitude (difference between maximum dilation and constriction of artery)

For statistical analysis ANOVA and t-test were used. $\mathrm{P}<0.05$ was considered as the level of significance. Results are given as mean $+/$ - standard error of the mean.

\section{RESULTS}

In a healthy individual, both retinal arteries and veins show a dilation in response to flickering light. After stimulation, the arterial as well as the venous diameter slowly return to baseline (Fig. 1a). Normal mean maximum arterial dilation is $6.9 \%+/-2.8$ s.d. in a healthy population.

In many glaucoma patients, the pattern of response to flickering light is different. As shown in the Fig. (1b) example there is no change or a small dilation in response to a flickering light.

The study group consisted of 26 POAG (primary open angle glaucoma) patients (10 female, 16 male) with a history of glaucoma of more than two years. Our patients were subdivided into two groups based on their preoperative response pattern. The 19 patients $(n=26 ; 73 \%)$ in group 1 preoperatively displayed an inadequate response to the flicker stimulation with a maximum arterial dilation of less than $4.0 \%$ despite a systemic acetazolamide therapy (see Methods). In group $2(\mathrm{n}=7 ; 27 \%)$, under systemic therapy with acetazolamide, the dilation of the arteries and veins to flickering light provocation did not differ significantly from those of healthy eyes.

Table 1 shows the patient characteristics of both groups: they did not differ significantly concerning age and blood pressure as well as pre- and postoperative intraocular pressure and initial baseline values (MU) of the DVA.

In both groups, there was a significant $(p<0.001)$ decrease of IOP at the time of the postoperative DVA measurement, from $21.6 \mathrm{~mm} \mathrm{Hg}$ to $9.0 \mathrm{~mm} \mathrm{Hg}$ in group 1 and from $20.2 \mathrm{~mm}$ $\mathrm{Hg}$ to $10.5 \mathrm{~mm} \mathrm{Hg}$ in group 2. DVA baseline values (MU) and diastolic and systolic blood pressure did not change significantly from pre- to postoperative.

Postoperatively, in group 1 there was a distinct improvement in the vessels' response compared to their behaviour before surgery. The median relative dilation of the arteries following the flicker provocation improved from $1.4 \%$ before to $3.8 \%$ following trabeculectomy $(\mathrm{p}<0.01)$. In retinal veins, this parameter increased from $3.1 \%$ to $4.6 \%(\mathrm{p}<0.05)$.

Despite this improvement, the postoperative values were still lower than the normal means and still significantly lower than those of postoperative group 2 patients (artery $\mathrm{p}<$ 0.001 ; vein $\mathrm{p}<0.05$ ). The peak amplitude of the retinal artery dilation increased slightly from 2.1 to $3.5 \%$, a difference that is not significant $(\mathrm{p}=0.09)$.

Fig. (1b) shows a typical example for a group 1 patient with a preoperatively reduced dilation answer of retinal vessels and with vascular spasms. After trabeculectomy the increase of vascular dilation response is showing typical changes for the better and a normalized vessel behaviour enabling an improved retinal perfusion at increased tissue demand (Fig. 1c).

In group 2 the vessel reaction did not differ significantly before and after trabeculectomy. The mean relative dilation of the arteries following the flicker stimulation changed nonsignificantly from $6.7 \%$ before to $6.3 \%$ following trabeculectomy; the venous response changed from $7.7 \%$ to $7.2 \%$ (ns). These values are within the normal range of a healthy population. 

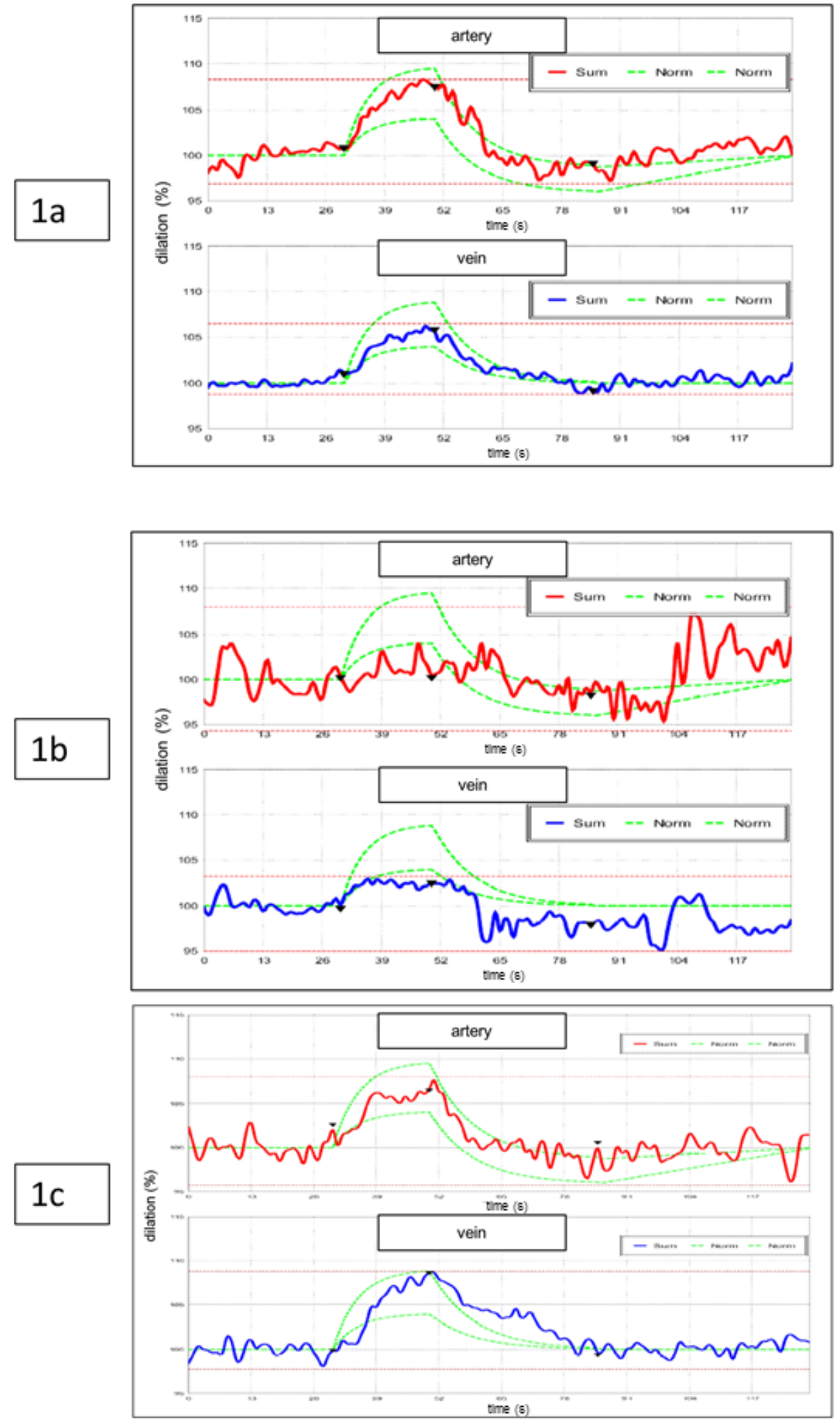

Fig. (1). (a) Typical behaviour of retinal vessels on flicker stimulation in a healthy eye as measured by Dynamic Vessel Analysis (red: artery; blue: vein). The first 30 seconds show uninfluenced baseline data. The average of uninfluenced vessel diameter measurements is set $100 \%$. Higher values indicate dilation and lower values contraction as compared to the baseline vessel diameters. At the following flicker light provocation vessel diameters dilate. After flicker stimulation they decrease and may even slightly fall below values at the starting level. After recovery they reach baseline values again. 'Sum' is giving all measurements of one patient in their temporal sequence. The area between the two 'Norm' curves represents the normal range. $(\mathbf{b}, \mathbf{c})$ Typical response of retinal vessels on flicker stimulation in a glaucomatous eye (group 1) as measured by Dynamic Vessel Analysis; (b) before trabeculectomy, (c) after trabeculectomy. A marked improvement of the vessel reaction can be seen. 
Table 1. DVA results before and after trabeculectomy. RR stands for blood pressure measurements according to Riva Rocci. IOP is the abbreviation for intraocular pressure assessed by Goldmann's applanation tonometry. RR and IOP are given in mm Hg. MU = measuring units, art = artery, ve $=$ vein.

\begin{tabular}{|c|c|c|c|c|c|}
\hline & $\begin{array}{c}\text { Group } 1 \\
\text { Mean }\end{array}$ & SEM & & $\begin{array}{c}\text { Group } 2 \\
\text { Mean }\end{array}$ & SEM \\
\hline $\mathbf{n}$ & 19 & & & 7 & \\
\hline age & 63.4 & 4.0 & ns & 60.0 & 5.1 \\
\hline RR syst & 125.5 & 4.0 & ns & 127.2 & 8.4 \\
\hline RR diast & 74.9 & 4.3 & ns & 79.6 & 3.3 \\
\hline \multicolumn{6}{|l|}{ before surgery: } \\
\hline IOP & 21.6 & 2.7 & ns & 20.2 & 2.4 \\
\hline baseline MU art & 106.6 & 4.0 & ns & 102.4 & 5.1 \\
\hline $\max$ dilation art $\%$ & 1.4 & 0.4 & $* *$ & 6.7 & 1.5 \\
\hline peak amplitude \% & 2.1 & 0.4 & $* *$ & 6.6 & 1.6 \\
\hline max constriction \% & -0.7 & 0.6 & ns & 0.1 & 0.3 \\
\hline baseline MU ve & 133.0 & 2.4 & ns & 127.8 & 7.2 \\
\hline max dilation ve $\%$ & 3.1 & 0.4 & $*$ & 7.7 & 1.8 \\
\hline \multicolumn{6}{|l|}{ after surgery: } \\
\hline IOP & 9.0 & 1.5 & ns & 10.5 & 1.5 \\
\hline baseline $\mathrm{MU}$ art & 109.5 & 4.2 & ns & 106.7 & 6.2 \\
\hline $\max$ dilation art $\%$ & 3.8 & 0.5 & $*$ & 6.3 & 0.5 \\
\hline peak amplitude $\%$ & 3.5 & 0.7 & $*$ & 7.4 & 1.8 \\
\hline max constriction \% & 0.5 & 0.5 & ns & -0.9 & 1.2 \\
\hline baseline MU ve & 138.6 & 2.6 & ns & 141.5 & 7.7 \\
\hline max dilation ve $\%$ & 4.6 & 0.6 & * & 7.2 & 1.0 \\
\hline
\end{tabular}

\section{DISCUSSION}

Our study demonstrates a positive effect of trabeculectomy, a typical IOP-lowering surgical procedure in glaucoma, on the function of retinal vessels as measured with dynamic vessel analysis (DVA).

Several devices have been developed over the years to evaluate retinal haemodynamics and come to a better understanding of its mechanisms [3]. None of these technologies has been able to cover the whole spectrum of retinal microcirculation, its physiology, and the pathological changes occurring in diseases like glaucoma or diabetic retinopathy.

Recently, Dynamic Vessel Analysis has emerged as a helpful tool in gaining insights into the dynamics of retinal circulation [13] and to determine its alterations in glaucoma a disease whose vascular aspects have become more evident in recent past.

Our study proves, for the first time, a beneficial effect of an IOP-lowering surgical intervention, standard trabeculectomy with Mitomycin $\mathrm{C}$ application, on the vascular response of those retinal vessels that had a diminished autoregulation before surgery (despite systemic acetazolamide!). In these patients with an inadequate response (group 1; 19 out of 26), DVA showed a significantly improved autoregulation after marked surgical IOP-lowering in both retinal arteries and in retinal veins.

In glaucoma patients without a preoperatively impaired autoregulation (group 2; 7 out of 26), there was no significant change in arterial and venous reaction to flickering light. The fact that these glaucoma patients demonstrate a normal vessel reaction before surgery might be an effect of the preoperatively administered acetazolamide. However, the postoperative measurements in these patients did show a normal vessel reaction comparable to that of healthy individuals although acetazolamide was stopped after surgery. Furthermore, the postoperative DVA measurements in this group 2 are significantly better than the postoperative values of group 1 . So it can be assumed that other unknown factors contribute to the effect in these patients. Further studies are necessary to characterize this group more precisely.

In other studies with glaucoma patients, we could show a similar beneficial effect on retinal vessels by lowering IOP with cyclophotocoagulation [16] whereas there was no effect on retinal haemodynamics despite pronounced IOP lowering of about $25 \%$ under a topical glaucoma therapy with latanoprost [17].

In the present study we were able to demonstrate the improvement in retinal vessel response to flickering light as 

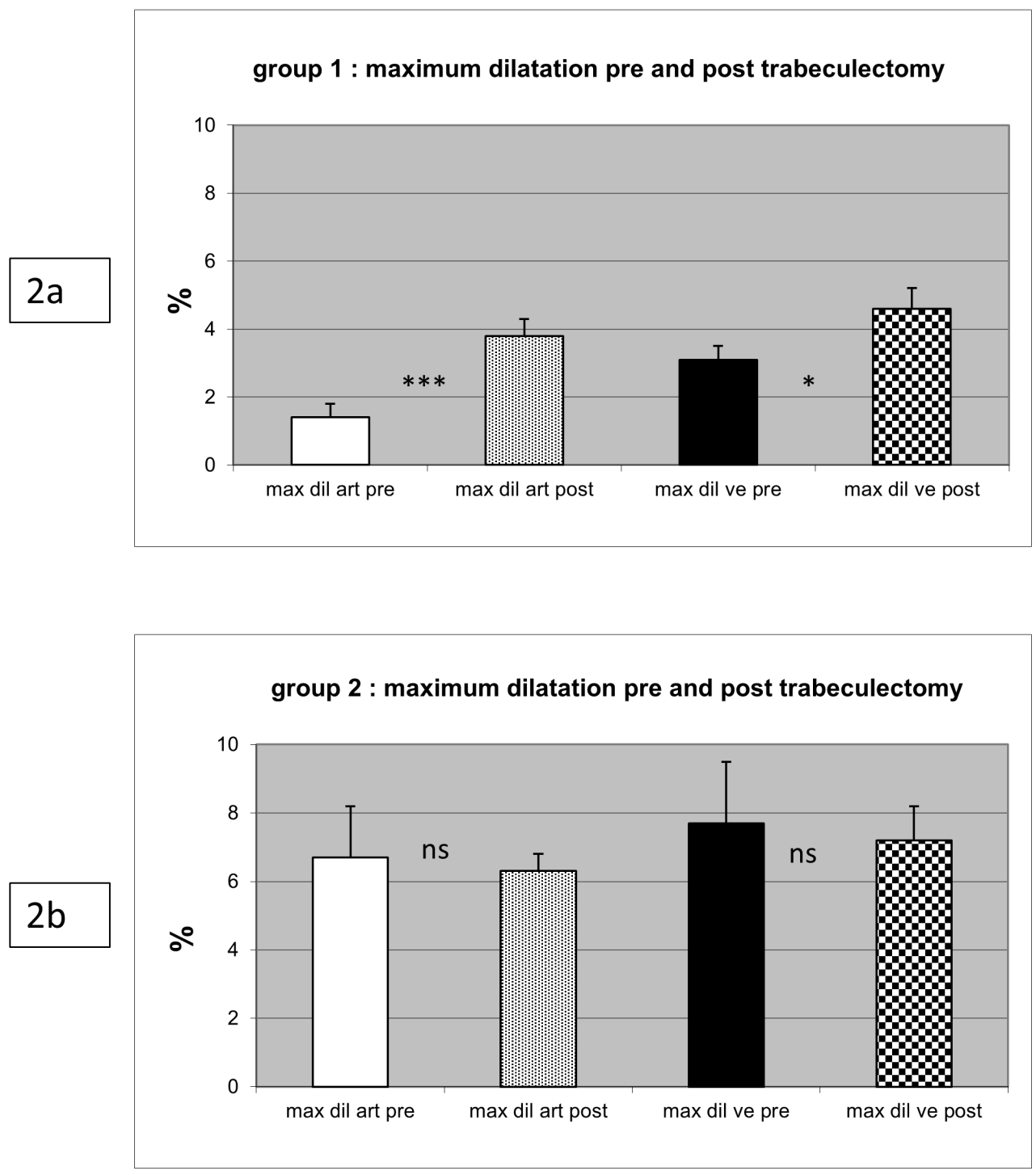

Fig. (2). Maximum dilation (\%) of artery and vein before and after trabeculectomy in group 1 and group 2 glaucoma patients after flicker stimulation. Mean +/- SEM. In group 1 a significant increase of the dilative response after trabeculectomy can be observed.

an indicator of the normalisation of ocular perfusion after surgical IOP lowering. The clinical implications of our study are evident: POAG patients with impaired vascular regulation might profit from IOP lowering trabeculectomy in improving vascular autoregulative reserve to compensate for blood pressure drop in the main circulation or for increases in the pulmonary circulation. Although the influence of glaucoma surgery on the oxygen saturation of these vessels has been ruled out by Hardarson et al. [18], there is, however, no doubt that the quantity of blood reaching the retina can be improved by surgery.

In this context DVA might be a helpful decision-making tool in favour of glaucoma surgery when a more profound IOP reduction is needed to enhance perfusion and thus to protect the optic nerve head from disease progression.

\section{CONFLICT OF INTEREST}

The authors declare that they have no conflict of interest. The only exception is Walthard Vilser who is CEO of IMEDOS Systems UG, Germany.

\section{ACKNOWLEDGEMENTS}

Supported by Ernst und Berta Grimmke-Stiftung, Vogelsanger Weg 49, 40470 Düsseldorf, Germany.

\section{REFERENCES}

[1] Flammer J. To what extent are vascular factors involved in the pathogenesis of glaucoma? In: Kaiser HJ, Flammer J, Hendrickson P, Eds. Ocular blood flow: new insights into the pathogenesis of ocular disease. Basel, Karger 1996; pp. 12-39.

[2] Drance SM. Disc haemorrhages in glaucoma. Surv Ophthalmol 1989; 33: 331-37.

[3] Caprioli J, Coleman AL. Blood pressure, perfusion pressure and glaucoma. Am J Ophthalmol 2010; 149: 704-12.

[4] Nagel E, Vilser W, Fink A, et al. Variance of retinal vessel diameter response to flicker light: a methodical clinical study. Ophthalmologe 2006; 103: 114-19.

[5] Nguyen TT, Kreis AJ, Kawasaki R, et al. Reproducibility of the retinal vascular response to flicker light in Asians. Curr Eye Res 2009; 12: 1082-88.

[6] Garhofer G, Bek T, Boehm A, et al. Use of the retinal vessel analyzer in ocular blood flow research. Acta Ophthalmol 2010; 88: $717-22$. 
[7] Heitmar R, Blann A, Cubbidge RP, et al. Continuous retinal vessel diameter measurements - the future of retinal vessel assessment? Invest Ophthalmol Vis Sci 2010; 51: 5833-39.

[8] Mandecka A, Dawczynski J, Blum M, et al. Influence of flickering light on the retinal vessels in diabetic patients. Diabetes Care 2007; 30: 3048-52.

[9] Schiel R, Vilser W, Kovar F, et al. Retinal vessel response to flicker light in children and adolescents with type 1 diabetes mellitus and overweight or obesity. Diabetes Res Clin Pract 2009; 83: 358-64.

[10] Lott ME, Slocomb JE, Shivkuma V, et al. Impaired vasodilator responses in prediabetes and type 2 diabetes. Acta Ophthalmol 2013; 91: 462-9.

[11] Mehlsen J, Jeppesen P, Erlandsen M, et al. Lack of effect of shortterm treatment with Amlodipine and Lisinopril on retinal autoregulation in normotensive patients with type 1 diabetes and mild diabetic retinopathy. Acta Ophthalmol 2011; 89: 764-8.

[12] Rickenbacher I, Gugleta K, Zawinka C, et al. Response of retinal vessel diameter to flicker-light in vasospastics compared to healthy controls. Klin Monatsbl Augenheilkd 2009; 226: 305-9.
[13] Garhofer G, Zawinka C, Resch H, et al. Response of retinal vessel diameters to flicker stimulation in patients with early open angle glaucoma. J Glaucoma 2004; 13: 340-44.

[14] Nagel N, Vilser W. Autoregulative behavior of retinal arteries and veins during changes of perfusion pressure: a clinical study. Graefe's Arch Clin Exp Ophthalmol 2004; 242: 19-17.

[15] Seifert BU, Vilser W. Retinal Vessel Analyzer (RVA) - Design and Function. Biomed Tech Suppl 2002; 1: 47.

[16] Kremmer S, Anastassiou G, Schallenberg M, et al. Laser Cyclophotocoagulation enhances the regulative capacity of retinal vessels in glaucoma. Open Ophthalmol J 2014; 8: 27-31 (accepted for publication).

[17] Kremmer S, Iliadou M, Anastassiou G, et al. Influence of Latanoprost on Retinal Microcirculation in Glaucoma. Open Ophthalmol J 2014; 8: 39-45.

[18] Hardarson S, Gottfredsdottir M, Halldorrson G, et al. Glaucoma filtration surgery and retinal oxygen saturation. Invest Ophthalmol Vis Sci 2009; 50: 5247-50.

(C) Selbach et al.; Licensee Bentham Open.

This is an open access article licensed under the terms of the Creative Commons Attribution Non-Commercial License (http://creativecommons.org/licenses/by-nc/3.0/) which permits unrestricted, non-commercial use, distribution and reproduction in any medium, provided the work is properly cited. 